\title{
INTESTINO DELGADO PATOLOGICO: RESONANCIA MAGNETICA CON CONTRASTE ORAL VERSUS TRANSITO INTESTINAL CON BARIO (RESULTADOS PRELIMINARES)*
}

Drs. Paula Csendes $G^{(1)}$, Claudio Cortés $A^{(1)}$, Claudio Silva $F^{(2)}$, Omar Enriquez $G^{(2)}$, Cristián Garrido ${ }^{(3)}$, Juan Maulen $A^{(3)}$.

1. Radiólogo 2. Residente Radiología 3. Tecnólogo Médico

Centro de Imagenología Hospital Clínico Universidad de Chile.

* Trabajo premiado por el comité de selección en el Congreso de Radiología 2004.

\begin{abstract}
Small bowel transit with barium sulphate allows adequate visualization of endoluminal alterations, but with drawbacks such as limited usefulness for wall compromise and extra parietal extension. Magnetic resonance appears to be an alternative, given its benefits avoiding radiation and allowing evaluation of anatomic details of the small bowel and adjacent structures.

Eleven patients with abnormal result of intestinal transit with barium underwent magnetic resonance with water 1500 cc p.o in brief period, with images achieved in a 1.5 Tesla resonator, with 25 $\mathrm{mT} / \mathrm{m}$ gradient, using optimized sequences.

Comparison in regards of time of transit, distension, and characterization of pathology was made.

An adequate correlation of the pathologic small bowel and its adjacent structures is achieved with magnetic resonance compared with transit with barium, achieving $90.9 \%$ of simple correlation and 0.82 Kappa value (excellent correlation), making this technique a valid and effective alternative to the conventional study of the small bowel with barium in our experience. Larger series are required to confirm this.
\end{abstract}

Key words: Magnetic resonance, Small bowel, Small bowel transit.

Resumen: El tránsito de intestino delgado con sulfato de bario permite una adecuada visualización de las alteraciones endoluminales, pero con limitaciones tales como no visualizar el compromiso y extensión extraparietal. La Resonancia Magnética aparece como una alternativa, con beneficios como evitar radiación y permitir la evaluación de detalles

Csendes P. y cols. Intestino delgado patológico: Resonancia magnética con contraste oral versus tránsito intestinal con bario (resultados preliminares). Rev Chil Radiol 2004; 10: 158-164.

Correspondencia: Dra. Paula Csendes G. Hospital Clínico Universidad de Chile. Santos Dumont 999 anatómicos de la pared intestinal y estructuras adyacentes.

En once pacientes con alteración del tránsito intestinal, se realizó resonancia magnética, usando agua en un volumen de 1500 cc como contraste oral administrado en un corto período de tiempo, obteniendo imágenes en resonador de 1.5 Tesla, con gradiente de $25 \mathrm{mT} / \mathrm{m}$. Las imágenes se compararon con estudio de intestino con bario para determinar tiempo de tránsito, parámetros de distensión y caracterización de la patología.

Una correlación de $90.9 \%$ y un valor Kappa de 0.82 (correlación excelente) del intestino delgado patológico y de las estructuras extraluminales adyacentes se logró con resonancia magnética, haciendo de esta técnica una alternativa válida y efectiva al estudio convencional del intestino delgado con bario en nuestra serie; sin embargo se requieren estudios con muestras mayores para confirmarla.

Palabras claves: Intestino Delgado, Resonancia Magnética, Tránsito Intestinal.

\section{Introducción}

La técnica de tránsito intestinal con sulfato de bario y rayos $X$, se ha utilizado tradicionalmente para el estudio de patología del intestino delgado, permitiendo una adecuada visualización de las alteraciones endoluminales ${ }^{(1)}$. Sin embargo, su utilidad en la pesquisa de lesiones de la pared, y especialmente la extensión extraparietal de ellas es limitada. La tomografía computada helicoidal ha sido una alternativa para obviar estas limitaciones ${ }^{(2,16)}$, pero mantiene el uso de radiación ionizante y el riesgo siempre presente de reacciones adversas producidas por el medio de contraste endovenoso.

Recientemente, se ha propuesto el uso de resonancia magnética (RM) para el estudio del intestino delgado, conocida sus ventajas de evitar la radiación ionizante y lograr información de detalles anatómicos, con excelente contraste tisular y capacidad de reconstrucciones multiplanares ${ }^{(3)}$. Para esra finalidad requiere de distensiones del lumen 
intestinal que solo se lograban con técnicas de enteroclisis, lo que implica un grado de invasión y molestia considerable para el paciente $\mathrm{e}^{(4,5,12,15,18,20)}$.

Algunas series con pequeño numero de casos han explorado la posibilidad de utilizar medios de contraste oral para el mismo propósito con resultados dispares ${ }^{(3,6,7,14,17,19,24-27)}$. Se ha propuesto la utilización de agua y el agregado a ella de agentes osmóticos para disminuir su reabsorción desde el lumen intestinal y tener una adecuada distensión ${ }^{(8,22,25)}$. Otra limitación técnica de este procedimiento consistía en los largos tiempos de adquisición de las imágenes, lo que introducía artefactos de movimiento respiratorio y por el peristaltismo. Actualmente, el desarrollo comercial de secuencias de gradiente rápidas han obviado estas limitantes, con una mayor aceptación del paciente y una mejor utilización del tiempo del resonador ${ }^{(9)}$.

Un estudio previo no publicado, nos demostró que es posible lograr niveles de distensión adecuados para obtener imágenes diagnósticas usando agua como medio de contraste y secuencias optimizadas para minimizar el problema de artefactos por peristaltismo y/o movimiento respiratorio. Dicha experiencia nos permitió además obtener información sobre las características de la pared intestinal en sujetos normales.

El propósito de este estudio es validar el agua como medio de contraste oral en resonancia magnética, correlacionando los hallazgos de exámenes patológicos de transito intestinal con bario y lo observado en RM de intestino delgado.

\section{Material y métodos}

En el período comprendido entre el 1 Enero y el 30 Junio del 2004, se reclutaron 11 pacientes que se realizaron tránsito de intestino delgado con bario y que presentaron alteraciones, siendo citados, previo consentimiento, en un plazo de 2 a 10 días para realizarse resonancia magnética de intestino delgado, con secuencias optimizadas producto de una línea de investigación previa.

Se administró 1,5 litros de agua fría en un período de 30 a 45 minutos. Posteriormente se realizó la adquisición de las imágenes en decúbito prono, para ejercer una suave presión en la pared anterior del abdomen, ordenando la distribución de las asas intestinales, disminuyendo los movimientos respiratorios y el volumen de la cavidad peritoneal y con ello una menor cantidad de cortes coronales.

Se utilizó resonador Siemens Magnetom Symphony 1.5 Tesla, con gradiente de $25 \mathrm{mT} / \mathrm{m}$ y bobina body array flex de 2 canales. En primer lugar se utilizó localizador echo gradiente en 3 planos (axial, sagital y coronal), realizándose cortes coronales con secuencias de gradiente rápidas potenciadas en T2 True FISP (True Fast Imaging with Steady-state Precession) y cortes axiales y sagitales en las áreas de interés. Estas secuencias se efectuaron con saturación grasa y sus parámetros se detallan en la tabla I. El tiempo de duración total de adquisición de las secuencias fue de 91 segundos, siendo el tiempo total del examen en el resonador de 10 minutos aproximadamente.

En el presente estudio se consideraron los efectos secundarios hasta 48 horas posteriores al examen. No se utilizaron fármacos antiespasmódicos u otras drogas.

Las adquisiciones fueron identificadas con un número aleatorio no conocido por quienes interpretaron las imágenes. Esta se realizó en forma independiente por dos radiólogos con experiencia en radiología digestiva (P.C; C.C.), quienes desconocían el resultado del estudio con bario. Se evaluaron parámetros de distensión, características de la pared intestinal, estructuras extraluminales y alteraciones anatómicas concomitantes.

Tabla I. Parámetros de las Secuencias de Pulso.

$\begin{array}{lccr}\text { Parámetro } & \begin{array}{l}\text { Localizador Echo } \\ \text { Gradiente }\end{array} & \text { TSE } & \text { True FISP } \\ \text { Grosor de Corte (mm) } & 8 & 60 & 5 \\ \text { Número de secciones de corte } & - & 2 & 30 \\ \text { Tiempo de Repetición (TR: mseg ) } & 11 & 4500 & 5,98 \\ \text { Tiempo de Echo (TE : mseg) } & 5,6 & 828 & 2,99 \\ \text { Field of View (FOV: mm }{ }^{2} \text { ) } & 500 & 350 & 350 \\ \text { Angulo Flip (Flip Angle) } & - & 120 & 70 \\ \text { Matriz } & 256 \times 144 & 448 \times 278 & 320 \times 214 \\ \text { Duración total (seg.) } & 25 & 18 & 25 \\ \text { Respiración } & \text { Normal } & \text { Apnea } & \text { Apnea }\end{array}$

(1) Nota: Se utilizó iPAT (Integrated Parallel Acquisition Techniques) en modo Grappa. 
Se consideró como distensión adecuada, aquella que permite visualizar en algunas de las fases de las adquisiciones, las asas no contraídas en segmentos de varios centímetros, lo que permite determinar el grosor de la pared intestinal entre dos asas contiguas.

\section{Resultados}

La población de estudio consistió en 11 sujetos, 4 hombres y 7 mujeres, con un promedio de edad de 45.7 años (rango: 19 a 75 años).

Hubo concordancia entre los hallazgos de RM y tránsito con bario en 10/11 pacientes (concordancia simple: $90.9 \%$ ), con un valor de concordancia kappa (Kappa-Bennet) de 0.82 , lo que corresponde a una correlación excelente o casi perfecta (en el rango: 0.8-1.0).

En el caso que no hubo concordancia se describe dilatación de asas intestinales y lenta progresión del bario en el estudio previo. Al momento de realizarse La RM 5 días después, el paciente presentaba una invaginación entero-enteral, la cual evolucionó con resolución espontánea.

Las patologías que se presentaron en el período de estudio fueron: 3 obstrucciones intestinales, 5 engrosamientos parietales focales, 2 invaginaciones y una malrotación intestinal (Tabla II).

Tabla II. Patologías estudiadas

- Malrotación

- Obstrucción intestinal

- Neoplasias intestino delgado

- Adenocarcinoma

- Adenoma

- GIST

- Invaginación enteroenteral

En los 11 casos se localiza la lesión en el mismo segmento por ambos estudios, con descripción similar de los hallazgos respecto a distensión de asas proximales y distales, proporcionando la RM información de estructuras adyacentes identificando en seis pacientes adenopatías mesentéricas, en dos engrosamientos del polo cecal, en dos reacciones inflamatorias del polo cecal, en uno una imagen sugerente de brida, en uno un quiste anexial y en un paciente colelitiasis.

Los tiempos de ingesta efectivos logrados fueron de 42 minutos en promedio (rango: 30-90 min), lográndose completar la ingesta total en menos de 60 minutos en el $90.9 \%$ de los casos.
Se constató que el tiempo de examen total, o para efectos prácticos, el tiempo de tránsito, fue en una sola adquisición en 10/11 pacientes (90.9\%), lo que determinó un tiempo de examen total, entre ingesta y adquisición promedio de 65 minutos (rango: 50-120).

En el seguimiento realizado, se obtuvo información de los 11 pacientes y los resultados se resumen en la tabla III. No se detectaron efectos adversos durante el periodo de hospitalización, ni en seguimiento telefónico posterior.

\section{Discusión}

El estudio del intestino delgado con el uso de sulfato de bario como medio de contraste se realiza desde 1927, y ha sido fundamental en el análisis de un segmento anatómico de difícil acceso por otros medios, incluidos los endoscópicos. Sin embargo, presenta limitaciones importantes ya que solo demuestra detalles de la mucosa y hay una superposición de las imágenes de asas intestinales. Existe además la posibilidad de floculación y/o segmentación de la columna de bario(10) y a veces un tiempo de examen prolongado; dado principalmente por la viscosidad del bario. Debe sumarse a ello el uso de radiación ionizante y la no menor incomodidad de la posterior eliminación del sulfato de bario, siendo necesario en ocasiones el uso de laxantes. Hasta la actualidad sigue siendo, sin embargo el "estandar dorado" imagenológico para el estudio del intestino delgado.

La RM surge como una aplicación de propiedades físicas, utilizadas en beneficio de las imágenes y optimizada con secuencias afines permite mejorar la resolución espacial en un sinnúmero de condiciones. Una gran ventaja es evitar el uso de radiación ionizante y de medios de contraste yodados endovenosos. Aplicada en patología intestinal, permite además la evaluación de estructuras extraparietales, mostrando la relación del intestino delgado con el resto de los órganos circundantes.

En el estudio de intestino, sin embargo, existía el problema de la presencia de movimientos dados por la respiración y el peristaltismo, lo que en secuencias del rango de un segundo, introducía elementos de borrosidad. Con la aparición de secuencias ultrarrápidas desarrolladas para otras aplicaciones, se abrió la posibilidad para su estudio.

Nuestra experiencia previa con RM y uso de agua como contraste oral nos demostró que se lograba una adecuada evaluación del intestino delgado y de las estructuras extraparietales. Usamos secuencias de gradiente rápidas potenciadas en T2 con medio de contraste vía oral evitando la enteroclisis. Solo nos faltaba evaluar esta técnica en casos de patología intestinal. 
Tabla III. Correlación radiológica con histología o hallazgos quirúrgicos.

\section{Paciente Imágenes (RM y Bario)}

$1 \quad$ Estenosis y obstrucción de yeyuno medio

2 Dilatación sacular del yeyuno, estenosis de anastomosis antigua

$3 \quad$ Masa sólida en yeyuno proximal, con dilatación proximal

$4 \quad$ Obstrucción del ileon, sugerente de brida

$5 \quad$ Malrotación intestinal

$6 \quad$ Invaginación enteroenteral

7 Engrosamiento mural de yeyuno

8 Masa de yeyuno con invaginación enteroenteral

9 Invaginación enteroenteral, imagen sugerente de lipoma

10 Engrosamiento del ileon compatible con enfermedad de Crohn

11 Engrosamiento segmentario del yeyuno ¿isquémico?

\section{Diagnóstico quirúrgico o histológico}

Adenocarcinoma de yeyuno

Estenosis anastomótica. Inflamación crónica inespecífica

GIST yeyuno proximal

Brida en ileon terminal, adherensiolisis

No hubo cirugía

Enfermedad celíaca (Biopsia)

Adenocarcinoma de yeyuno

Adenoma de yeyuno alto

Lipoma de ileon proximal

Enfermedad de Crohn

No hubo cirugía
En este estudio demostramos que existe una muy buena correlación entre los hallazgos del tránsito intestinal con bario y la RM de intestino delgado, permitiendo además información importante respecto a las estructuras adyacentes, como por ejemplo, presencia de adenopatías, y algunas características parietales. Por otra parte, el tiempo total de examen o tiempo de tránsito con un valor máximo de alrededor de 90 minutos en total es breve comparado al estudio convencional con sulfato de bario. Tenemos que hacer notar que la utilización de equipo fue de alrededor de 60 minutos en un $90.9 \%$ de los casos. Esto permite considerar la optimización del tiempo, ya que permite realizar varios estudios en forma seriada o intercalar exámenes breves entre adquisiciones de intestino delgado.

Existen publicaciones, respecto a la utilización de $\mathrm{RM}$ en patologías como enfermedad de Crohn ${ }^{(5,11,12,15)}$ y algunos reportes de casos en otras patologías como carcinoide ${ }^{(13)}$, obstrucción intestinal| ${ }^{(21,29)}$ y enfermedad celíaca ${ }^{(17)}$, sin embargo, ellos requirieron el uso de enteroclisis para lograr imágenes diagnósticas, lo que implica una importante incomodidad para el paciente. Hasta nuestro conocimiento, no existe experiencia comunicada en nuestro medio con el uso de nuestra técnica de examen.

\section{Conclusión}

Nuestro protocolo con contraste vía oral y secuencias rápidas de gradiente potenciadas en T2, ha permitido determinar su utilidad en análisis de intestino delgado sano. En el presente estudio hemos demostrado su aplicabilidad en variados tipos de patología intestinal. El mayor costo de RM, puede ser una limitante para ser usada como estudio inicial, sin embargo, considerando la información anatómica que aporta con una buena resolución, debe evaluarse elementos de costo-efectividad. 
Figura 1 a-d. Adenoma de yeyuno alto. a: Tránsito intestinal baritado demostrando la lesión como un defecto de Ilenamiento intraluminal (flechas). b: Placa localizada c: RM secuencia True FISP coronal demostrando claramente la lesión (A). d: Pieza quirúrgica.

Figura 2 a,b. Enfermedad de Crohn del ileon. Se identifica con flechas el asa comprometida a: Tránsito intestino delgado baritado demostrando los signos clásicos. $\boldsymbol{b}$ : RM secuencia True FISP coronal demostrando el compromiso del lumen y la pared. 
Figura 3 a-c. Paciente con enfermedad celíaca en quien se demostró una invaginación entero-enteral en el momento de efectuar la RM. a: Tránsito intestino delgado baritado. b,c: RM secuencia True FISP coronal. Se identifica con flechas la invaginación en $\boldsymbol{c}$.

Figura 4 a,b. Malrotación intestinal con las asas de delgado ocupando el flanco derecho. a: Tránsito intestino delgado baritado. b: RM secuencia True FISP coronal.

Figura 5. Obstrucción intestinal por bridas. a: Tránsito intestino delgado baritado. b: RM secuencia True FISP coronal.

\section{Bibliografía}

1. Notan DJ, Trail ZC. The current role of the barium examination of the small intestine. Clin Radiol 1997; 52: 809-20.

2. Raptopoulos V, Schwartz RK, McNicholas MMJ. et al. Multiplanar helical CT enterography in patients with Crohn's disease. Am J Roentgenol AJR 1997; 169: 1545-50. 
the Small Bowel Using Water as a Contrast Agent in a Preliminary Study with Healthy Volunteers. Am J Roengenol AJR 1999; 173: 581-582.

7. Patak MA, Froehlich JM, von Weymarn C. et al. Non invasive distension of the small bowel for magneticresonance imaging. Lancet 2001; 358: 987-88.

8. Lauenstein TC, Schneemann H, Vogt FM. et al. Optimization of Oral Contrast Agents for MR Imaging of the Small Bowel. Radiology 2003; 228: 279-283.

9. Keogan MT, Edelman RR. Technologic Advances in Abdominal MR Imaging, State of the Art. Radiology 2001; 220: 310-320.

10. Freeman $\mathrm{AH}$, Barltrop $\mathrm{AH}$. Limitations of barium small bowel studies. Imaging 1999; 11: 139-143.

11. Narin B., Ajaj W., Gohde S. et al. Combined small and large bowel MR imaging in patients with Crohn's disease: a feasibility study. Eur Radiol. 2004; 14(9): 1535-42.

12. Gourtsoyiannis N, Papanikolaou N, Grammatikakis $J$. et al. Assessment of Crohn's disease activity in the small bowel with $\mathrm{MR}$ and conventional enteroclysis: preliminary results. Eur Radiol. 2004; 14(6):1017-24.

13. Azoulay R, Boudiaf M, Soyer P. et al. [Carcinoid tumor of the small bowel: value of hydro-MR imaging for diagnosis] J Radiol. 2003; 84(12 Pt 1): 1982-5. (Abstract)

14. Ajaj W, Goehde SC, Schneemann H. et al. Oral contrast agents for small bowel MRI: comparison of different additives to optimize bowel distension. Eur Radiol. 2004; 14(3): 458-64.

15. Broglia L, Gigante P, Papi C. Et al. Magnetic Resonance enteroclysis imaging in Crohn's disease. Radiol Med (Torino). 2003; 106(1-2):28-35. (Abstract)

16. Schmidt S, Lepori D, Meuwly JY. et al. Prospective comparison of MR enteroclysis with multidetector spiral-CT enteroclysis: interobserver agreement and sensitivity by means of «sign-by-sign» correlation. Eur Radiol. 2003; 13(6): 1303-11.

17. Laghi A, Paolantonio $P$, Catalano C. et al. MR imaging of the small bowel using polyethylene glycol solution as an oral contrast agent in adults and children with celiac disease: preliminary observations. Am J Roentgenol AJR 2003; 180(1): 191-4.

18. Gourtsoyiannis N, Papanikolaou N, Grammatikakis J. et al. MR enteroclysis: technical considerations and clinical applications. Eur Radiol. 2002; 12(11): 2651-8.

19. Laghi A, Carbone I, Paolantonio P. et al. Polyethylene glycol solution as an oral contrast agent for MR imaging of the small bowel. Acad Radiol. 2002; 9 Suppl 2: S355-6.

20. Papanikolaou N, Prassopoulos P, Grammatikakis J. et al. Optimization of a contrast medium suitable for conventional enteroclysis, MR enteroclysis, and virtual MR enteroscopy. Abdom Imaging. 2002; 27(5): 517-22.

21. Beall DP, Fortman BJ, Lawler BC, Regan F. Imaging bowel obstruction: a comparison between fast magnetic resonance imaging and helical computed tomography. Clin Radiol. 2002; 57(8): 719-24.

22. Minowa O, OzakiY, Sumi Y. [MR imaging of small bowel with water administration] Nippon Igaku Hoshasen Gakkai Zasshi. 2002; 62(2): 73-8. (Abstract)

23. Kim JH, Ha HK, Sohn MJ. Et al. Usefulness of MR imaging for diseases of the small intestine: comparison with CT. Korean J Radiol. 2000; 1(1): 43-50.

24. Laghi A, Carbone I, Catalano C. et al. Polyethylene glycol solution as an oral contrast agent for MR imaging of the small bowel. AJR Am J Roentgenol 2001; 177(6): 1333-4.

25. Gourtsoyiannis N, Papanikolaou N, Grammatikakis J. et al. MR imaging of the small bowel with a true-FISP sequence after enteroclysis with water solution. Invest Radiol 2000; 35(12): 707-11.

26. Rieber A, Aschoff A, Nussle K. et al. MRI in the diagnosis of small bowel disease: use of positive and negative oral contrast media in combination with enteroclysis. Eur Radiol 2000; 10(9): 1377-82.

27. Umschaden HW, Szolar D, Gasser J. et al. Small-bowel disease: comparison of MR enteroclysis images with conventional enteroclysis and surgical findings. Radiology 2000; 215(3): 717-25.

28. Grubnic S, Padhani AR, Revell PB, Husband JE. Comparative efficacy of and sequence choice for two oral contrast agents used during MR imaging. Am J Roentgenol AJR 1999; 173(1): 173-8.

29. Regan F, Beall DP, Bohlman ME. et al. Fast MR imaging and the detection of small-bowel obstruction. Am J Roentgenol AJR 1998; 170(6):1465-9.

30. Anderson CM, Brown JJ, Balfe DM et al. MR imaging of Crohn disease: use of perflubron as a gastrointestinal contrast agent. J Magn Reson Imaging 1994; 4: 491-6. 